\title{
High-speed and broadband optical transmission system special issue based on selected IEEE ICOCN 2015 papers
}

\author{
Shilong Pan ${ }^{1} \cdot$ Alan Pak Tao Lau ${ }^{2} \cdot \mathrm{Lei} \mathrm{Guo}^{3} \cdot \mathrm{Hoon} \mathrm{Kim}^{4}$
}

Published online: 21 September 2016

(C) Springer Science+Business Media New York 2016

The 14th International Conference on Optical Communications and Networks-IEEE ICOCN 2015 (http://icocn2015. nuaa.edu.cn) was held in Nanjing on July 3-5, 2015.

IEEE ICOCN aims to provide a premier opportunity for professionals, experts, engineers, scientists, and industrial people worldwide in the field of research, development and applications of photonics to share and exchange their experience. IEEE ICOCN 2015 included four technical tracks: (1) photonic devices and integration, (2) optical networks and transmission systems, (3) microwave photonics and analog optical systems, and (4) fiber optics, fiber devices and optical sensing.

IEEE ICOCN 2015 was a 3-day event organized by Nanjing University of Aeronautics and Astronautics, Nanjing University and Nanjing University of Posts and Telecommunications, and was technically sponsored by IEEE Photonics Society. The conference was also supported by KEYSIGHT Technologies, ROHDE \& SCHWARZ, Jiangsu Society of Aeronautics and Astronautics, Nanjing Society of Optical Communications and Photonic Technology, Luster LightTech Group, and Conquer Company.

We accepted more than 217 papers by the authors from more than 18 countries and regions, among which 70 are invited talks. All the papers were carefully reviewed by more than 60 Technical Program Committee (TPC) members and

Shilong Pan

pans@nuaa.edu.cn

1 Nanjing University of Aeronautics and Astronautics, Nanjing, People's Republic of China

2 Hong Kong Polytechnic University, Kowloon, Hong Kong

3 Northeastern University, Shenyang, People's Republic of China

4 Korea Advanced Institute of Science and Technology, Daejeon, Korea external reviewers, with each paper evaluated by at least two reviewers. All these papers and talks are organized into eight lecture-style oral sessions and one interactive poster session.

Based on the comments received from the reviewers, the authors of 14 accepted papers that are in the area of HighSpeed and Broadband Optical Transmission System were invited to submit an extended version of their work for possible publication in this Special Issue of the Springer Photonic Network Communications (PNET) journal. After a thorough review process, 13 papers have been selected for publication. In addition, this Special Issue includes invited papers that went through the same review process of the other accepted papers. A brief summary on the accepted papers is provided next.

In "Visible light communication application scenarios based on android smart devices' led lamp," C. Wang, Z. Zhang, M. Zhang, and H. Zhu propose two different Android mechanisms to make the flicker in different data rate by using LED lamps as the transmitter. By optimizing the code framework and using new function in the hardware driver layer, the operating efficiency can be increased greatly, a maximum data rate of $5000 \mathrm{bps}$ is achieved on an Android development board.

In "Q-band radio-over-fiber system for distribution of uncompressed high-definition video signal," Z. Z. Tang and S. L. Pan propose a Q-band RoF system for the distribution of uncompressed HD video signal. Q-band (40.5-47 GHz) is suggested for short-range communication in China, and a protocol named IEEE 802.11aj will be released in 2016. The huge unlicensed bandwidth $(5.9 \mathrm{GHz})$ coupled with moderate transmit power and rapid advances in integrated circuit (IC) technologies makes Q-band a promising candidate for the high data rate wireless communication.

In "Application of local error method to SSSF simulation of vector propagation in dispersion compensated optical 
links," Q. Zhang, M. I. Hayee, V. Winstead, S. Nadimpalli, X. Wu, D. Huang, L. Jie, and M. Khaliq demonstrate the scaling of the global simulation error with respect to the number of optical fiber spans and global error control for multi-span simulations. Combining the local error and global error control, the developed simulation scheme can significantly speed up the time consuming simulations in coherent optical fiber communication system analysis and design.

In "Optical time-division multiplexing signal processing using electro-optic modulators," L. Huo, D. Wang, Q. Wang, Y. F. Xing, X. Y. Jiang and C. Y. Lou present some optical signal processing methods for optical timedivision multiplexing using electro-optic modulators. Timelens assisted-processing and dual-parallel Mach-Zehnder modulator-based processing for optical frequency-comb generation, optical Nyquist pulse generation, equivalent switching window narrowing for demultiplexing are realized. Simultaneous clock recovery and demultiplexing using modified configurations based on optoelectronic oscillators are demonstrated.

In "Flexible decision-aided maximum likelihood phase estimation in coherent optical phase-shift-keying systems," X. D. You, J. Chen, P. Wang, C. Y. Yu, and P. F. Li take into account the time-varying laser phase noise and propose a flexible Decision-aided maximum likelihood (DA-ML) phase estimation method for carrier phase recovery in coherent optical phase-shift-keying systems so as to eliminate BLE. Weighted coefficients based on ML criterion are introduced to strengthen the estimation accuracy. The statistical property of phase estimation error is derived, and the bit error rate (BER) performance is also evaluated.

In "Nonlinear pulse shaping-assisted ultra-short optical pulse generation," D. Wang, W. K. Yu, L. Huo, X. Y. Jiang, X. Chen, and C. Y. Lou propose and demonstrate an ultra-short optical pulse generator based on nonlinear pulse shaping. Nearly, chirp-free 2.2-ps optical Gaussian pulse and 0.73-ps optical Nyquist pulse with a repetition rate of $25 \mathrm{GHz}$ are achieved in simulation.

In "Performance comparision of DA-TDE and CMA for MIMO equalization in multimode multiplexing systems," $\mathrm{X}$. Xiang, Y. Li, C. R. Tu, J. Wu, W. Li, and J. T. Lin compare performances of the constant modulus algorithm (CMA) and data-aided time domain equalization (DA-TDE) algorithm for mode demultiplexing in few-mode transmission systems. Simulation results of $4 \times 56 \mathrm{Gbit} / \mathrm{s}$ QPSK mode-division multiplexed system show that the maximum required optical signal to noise ratios (OSNRs) with CMA are less than that of DA-TDE by 0.8 and $4.6 \mathrm{~dB}$ for high maximum coupling coefficient of 0.15 and 0.20 .

In "All-fiber sensor based on few-mode fiber offset splicing structure cascaded with long-period fiber grating for curvature and acoustic measurement," X. Fu, P. Lu, C. Luo, J. Chen, S. Wang, and C. Zhang report a sensor for curvature and acoustic sensing based on a single-mode-few-modesingle-mode fiber offset splicing structure cascaded with long-period fiber grating and analyze the spectral characteristic and sensing mechanism. In order to demodulate the curvature, the intensity demodulation is adopted to measure the change of optical transmission power of a certain wavelength.

In "Post-FEC performance evaluation of coherent QPSK system with an Enhanced Pilot-Aided CPE Scheme," H. Q. Cheng, H. Z. Chen, Y. Li, C. Lu, J. Wu, and J. T. Lin propose a simple and robust pilot-aided carrier phase estimation scheme with a modified phase unwrapping. The principle of the proposed phase unwrapping is shown with a step by step procedure. They also test the post-FEC bit error rate (BER) performance for the pilot-aided carrier phase estimation scheme with the traditional and proposed phase unwrapping.

In "Digital signal processing techniques in Nyquist-WDM transmission systems," Z. Q. Pan, J. Y. Wang, and Y. Weng introduce the background of digital signal processing for Nyquist spectral shaping in optical fiber WDM systems. They investigated the use of digital finite impulse response (FIR) filters to generate Nyquist-WDM 16-ary quadrature amplitude modulation (16QAM) signals with the raised-cosine (RC) and root-raised-cosine (RRC) shape spectrum. The system performance of both the RC and RRC spectra is also examined.

In "Modulation format identification in heterogeneous fiber-optic networks using artificial neural networks and genetic algorithms," S. T. Zhang, Y. Q. Peng, Q. Sui, J. P. Li, and Z. H. Li propose the use of genetic algorithm (GA) to simplify the structures of artificial neural network (ANN)-based format identification (MFI) techniques to realize MFI in next generation dynamic and heterogeneous fiber-optic networks and enhance the efficiency of optical performance monitoring (OPM). Simulation results demonstrate significant improvement in MFI performance compared with conventional ANN techniques.

In "An effective carrier phase estimation scheme in faster than Nyquist-WDM transmission system," D. W. Pan, C. C. Li, Y. Q. Feng, and X. G. Zhang propose a carrier phase estimation method using unequaled digital pilot-tone in a faster than Nyquist-WDM transmission system, in which the digital pilot-tone is employed to estimate laser phase noise induced by the lasers located at the transmitter (Tx) and at the receiver ( $\mathrm{Rx}$ ) ends. The method can effectively overcome the scale of the inter-symbol interference (ISI) introduced by faster than Nyquist (FTN) signaling, which also shows features of high phase noise tolerance and simple hardware complexity.

In "Dither-free low-bias controller for deeply modulated Mach-Zehnder modulators," J. Y. Ning, Y. T. Dai, F. F. Yin, J. Q. Li, J. Y. Zhang, Y. Zhou, and Kun Xu propose and exper- 
imentally demonstrate a novel dither-free low-bias control technique especially for deeply modulated analog photonic links. In our scheme, a second lightwave passes through the MZM, while the output power is locked. Since the reverse modulation efficiency is very low when the input radio frequency (RF) carrier is as high as $\mathrm{GHz}$, the RF power shows little impact on the optical power even the bias angle is very low, so that the bias stabilization is achieved.

\section{IEEE ICOCN 2015 General TPC Chair}

Shilong Pan, Nanjing University of Aeronautics and Astronautics, P. R.China, pans@ nuaa.edu.cn

\section{IEEE ICOCN 2015 Transmission Subcommittee Co-Chairs}

Alan Pak Tao Lau, Hong Kong Polytechnic University, Hong Kong, alan.pt.lau@polyu.edu.hk

Lei Guo, Northeastern University, P. R. China, haveball@163.com

Hoon Kim, Korea Advanced Institute of Science and Technology, Korea, hoonkim@kaist.ac.kr

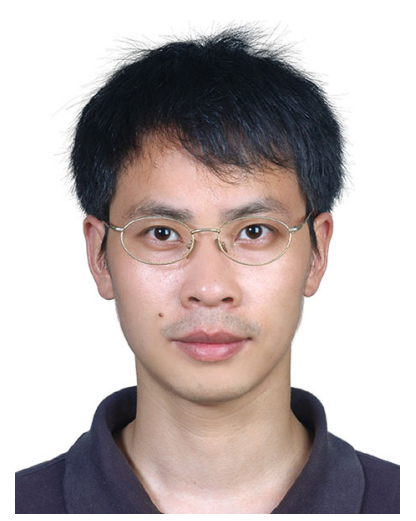

Shilong Pan (S'06-M'09SM'13) received the B.S. and Ph.D. degrees in electronics engineering from Tsinghua University, Beijing, China, in 2004 and 2008, respectively. From 2008 to 2010, he was a "Vision 2010" Postdoctoral Research Fellow in the Microwave Photonics Research Laboratory, University of Ottawa, Canada. He joined the College of Electronic and Information Engineering, Nanjing University of Aeronautics and Astronautics, China, in 2010, where he is currently a full professor and executive director of the Key Laboratory of Radar Imaging and Microwave Photonics (Nanjing Univ. Aeronaut. Astronaut.), Ministry of Education. His research has focused on microwave photonics, which includes optical generation and processing of microwave signals, analog photonic links, photonic microwave measurement, and integrated microwave photonics. Prof. Pan has authored or co-authored over 250 research papers, Including more than 130 papers in peer-reviewed journals and 120 papers in conference proceedings. Prof. Pan serves as a Chair of numerous international conferences and workshops, including the TPC Chair of the International Conference on Optical Communications and Networks in 2015, TPC Chair of the high-speed and broadband wireless technologies subcommittee of the IEEE Radio Wireless Symposium in 2013, 2014, and 2016, TPC Chair of the Optical fiber sensors and microwave photonics subcommittee of the OptoElectronics and Communication Conference in 2015, and Chair of the microwave photonics for broadband measurement workshop of International Microwave Symposium in 2015. He also serves as a Topical Editor of Chinese Optics Letters.

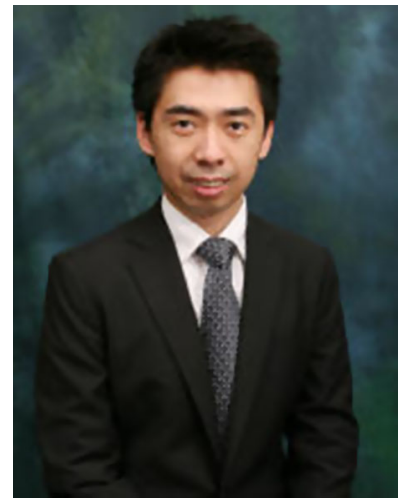

Alan Pak Tao Lau received his B.A.Sc in Engineering Science (Electrical Option) and M.A.Sc. in Electrical and Computer Engineering from University of Toronto in 2003 and 2004 respectively. He obtained his Ph.D. in Electrical Engineering at Stanford University in 2008 and has joined the Hong Kong Polytechnic University as an Assistant Professor and. $\mathrm{He}$ worked at NEC Labs America in summer 2006 on receiver structures for multi-mode fiber systems. He is now an Associate Professor, and his current research interests include various aspects of coherent fiber-optic communication.

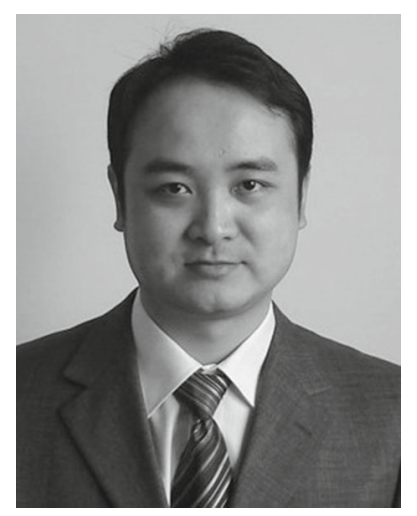

Lei Guo received the Ph.D. degree in communication and information systems from School of Communication and Information Engineering, University of Electronic Science and Technology of China, Chengdu, China, in 2006. He is currently a professor in School of Computer Science and Engineering, Northeastern University, Shenyang, China. His research interests include optical networks, wireless networks and converged optical-wireless networks. He has published over 200 technical papers in the above areas. Dr. Guo is a member of IEEE and OSA. He was the recipient of the Best Paper Award from ICCCAS'04. He is currently servicing as the Editorial Board Member of The Open Optics Journal and the International Journal of Digital Content Technology and its Applications.

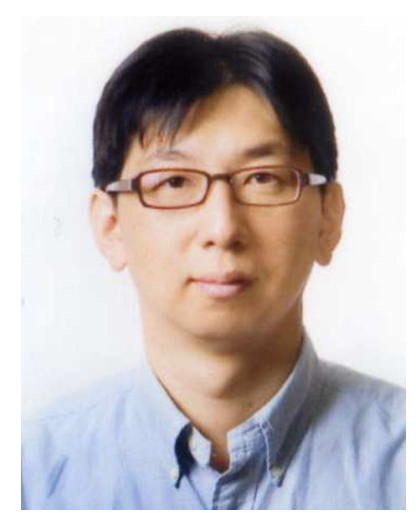

Hoon Kim is an Associate Professor of the School of Electrical Engineering at Korea Advanced Institute of Science and Technology (KAIST). Prior to joining KAIST in 2014, he was with Bell Labs, Lucent Technologies (2000-2001), Samsung Electronics, Korea (2001-2007), and National University of Singapore (2007-2014). He currently serves as Associate Editors of IEEE Photonics Technology Letters and Optics Express. His research interests include highcapacity fiber-optic communication systems, broadband optical access networks, mobile fronthaul/backhaul networks, and lightwave subsystems. 